\title{
Surgical Site Infection Prophylaxis and Wound Management in Spine Surgery
}

\author{
Alexandra J. White ${ }^{1}$, Brian Fiani ${ }^{2}$, Ryan Jarrah ${ }^{3}$, Arbaz A. Momin ${ }^{1}$, Jonathan Rasouli ${ }^{4}$ \\ ${ }^{1}$ Cleveland Clinic Lerner College of Medicine of Case Western Reserve University, Cleveland, OH, USA \\ ${ }^{2}$ Department of Neurosurgery, Desert Regional Medical Center, Palm Springs, CA, USA \\ ${ }^{3}$ Department of Neurosurgery, Mayo Clinic, Rochester, MN, USA \\ ${ }^{4}$ Department of Neurological Surgery, Cleveland Clinic, Cleveland, OH, USA
}

Surgical site infection (SSI) is a potentially devastating complication of spinal surgery that increases patient morbidity and healthcare costs. SSIs have complex and multifactorial etiologies; therefore, there are numerous opportunities for prevention and risk mitigation. The aim of this narrative review was to describe the incidence, risk factors, and outcomes of SSIs in spine surgery with an emphasis on postoperative wound care. We list and describe the preoperative, intraoperative, and postoperative evidence-based interventions that can be applied to potentially prevent SSI after spinal surgery.

Keywords: Spine; Surgical wound infection; Intraoperative care; Postoperative care; Perioperative care

\section{Introduction}

Surgical site infections (SSIs) are the third most common complication after spine surgery, following pneumonia and urinary tract infection (UTI) [1-3]. A 2019 metaanalysis reported a pooled SSI incidence of $3.1 \%$ among 22,475 patients, with a $1.4 \%$ incidence of superficial SSI and a $1.7 \%$ incidence of deep SSI [4]. The highest incidence (13\%) was reported in patients with neuromuscular scoliosis [4]. The most common pathogens responsible for SSI in spine surgery are Staphylococcus aureus (37.9\%), Staphylococcus epidermidis (22.7\%), and methicillin-resistant Staphylococcus species (23.1\%) [4]. SSIs are reported to increase the length of hospital stay and mortality [5]. SSIs may require further revision surgeries or lead to chronic pain or deformity [6,7]. Infection, including SSI, deep space infection, and bacteremia, was the most common (28.2\%) cause of 30-day readmission following spinal surgery in a 2015 meta-analysis [8]. However, a 2015 retrospective study of infections after dorsal cervical spinal surgery showed that while patients with SSI reported a compromised quality of life in the short term compared to controls, this difference disappeared at 1 year after the surgery, suggesting that these detrimental effects may be temporary [7].

\section{Costs}

Multiple studies have documented a relationship between SSI and increased cost of treatment [7,9-11]. Methicillin-

Received Feb 22, 2021; Revised Feb 22, 2021; Accepted Mar 11, 2021

Corresponding author: Jonathan Rasouli

Department of Neurological Surgery, Cleveland Clinic, Cleveland, OH, USA

Tel: +1-440-312-6100, Fax: +1-444-312-0454, E-mail: rasoulj@ccf.org 
resistant Staphylococcus aureus (MRSA) infection, increased number of fused levels, and intensive care unit stay are associated with increased healthcare costs of readmission for SSI [11]. It is noteworthy that interventions, such as the use of topical vancomycin powder, are known to lower costs, resulting in savings of $\$ 433,765$ per 100 posterior spinal fusions [12]. The implementation of protocolized infection prophylaxis measures has produced encouraging results in terms of both, reduced infection rates and surgery-related costs, suggesting that a systematic approach for preventing SSI in spine surgery is most effective [13].

\section{Risk Factors}

The etiology of SSI after spinal surgery is complex and multifactorial. Pre-procedural risk factors include diabetes, male sex, obesity, hypertension, chronic obstructive pulmonary disease, UTI, prior surgery, American Society of Anesthesiology (ASA) score $>3$, and prolonged corticosteroid use [4,6,14-20]. ASA score serves as a proxy for frailty; age; and chronic illnesses, such as diabetes. A 2013 meta-analysis found that when controlling for confounders, such as diabetes, a 5-point increase in body mass index (BMI) led to a 21\% increase in the SSI incidence [19].

Diabetes mellitus (both types 1 and 2) is also a major risk factor for SSI after spine surgery $[20,21]$. The risk is higher in patients with insulin-dependent diabetes than in those with diabetes that is controlled with diet or with oral medications [5]. Preoperative serum glucose of $>125$ $\mathrm{mg} / \mathrm{dL}$ or postoperative serum glucose $>200 \mathrm{mg} / \mathrm{dL}$ is associated with an increased risk of infection in patients undergoing orthopedic spinal surgery, suggesting that optimizing glycemic control preoperatively is an infectionprevention strategy [22]. Diabetes leads to immunocompromise and impairs microvascularization and wound healing potential $[5,14]$. Coronary artery disease has also been associated with SSI in spine surgery [16,20]. As such, risk factor mitigation for SSI in elective spine surgery patients should begin considerably before the date of surgery and includes smoking cessation, glycemic control, and optimum management of chronic disease.

Operative indication also influences the risk of SSI, with increased risk of SSI being reported in surgery for acute osteomyelitis/discitis, deformity, trauma, or kyphosis in the pediatric population $[2,21,23]$. Trauma may increase the risk of SSI because of tissue hypoxia and soft tissue injury [3]. Cervical spine surgery has an SSI incidence of $3.4 \%$, thoracic spine surgery has an SSI incidence of $3.7 \%$, and lumbar spine surgery has an incidence of $2.7 \%$ [4].

The risk of SSI varies with the surgical approach. The incidence was higher in patients for whom surgery was performed with instrumentation (4.4\% versus $1.44 \%$ ) and with allograft use, likely due to the introduction of foreign material into the wound $[3,4,16]$. Surgery at academic medical centers has also been identified as an independent risk factor for deep SSI in posterior instrumented fusion [18]. Minimally invasive techniques are associated with a reduced incidence of SSI than open surgery $[4,24]$. This is corroborated by the reported association between the incision length and increased infection risk [3]. Fusion at a higher number of levels is also associated with SSI $[22,25]$.

Certain intraoperative complications, such as dural tears, can increase the SSI risk [6]. Dural tears allow communication between the epidural space and the cerebrospinal fluid (CSF). They prolong the duration of surgery and length of postoperative bed rest $[6,15]$. Moreover, the need for blood transfusion is an independent risk factor for SSI in spine surgery [16].

\section{Preoperative Interventions}

\section{Nasal Staphylococcus aureus culture}

Colonization of the nares by both Methicillin-sensitive Staphylococcus aureus (MSSA) and MRSA has been described as a risk factor for SSI development. A 2020 metaanalysis by Ning et al. [26] of MSSA and MRSA colonization in patients undergoing spinal surgery reported $18.8 \%-35.6 \%$ prevalence of overall S. aureus colonization and $1.1 \%-5.9 \%$ prevalence of MRSA colonization. While MSSA colonization was not associated with an increased risk of SSI in this study, Higgins and colleagues reported that MSSA screening before the surgery was related to a higher risk of SSI and lowered the rates of SSI; therefore, screening for MSSA remains controversial [27].

In contrast, MRSA colonization was associated with a significantly increased risk of both, MRSA and overall SSI [26]. This relationship disappeared when the patients were prescribed topical muciprocin ointment before the surgery for decolonization of the nares $[26,28]$. Therefore, reverse transcription polymerase chain reaction screening for MRSA colonization should occur at least 5 days preoperatively, followed by treatment with $2 \%$ muciprocin 
ointment and showers with chlorhexidine soap. This approach was demonstrated to reduce the rate of SSI by $>50 \%$ in a 2010 randomized controlled trial [29]. Furthermore, MRSA decolonization should be confirmed with a swab prior before the surgery $[26,28]$.

\section{Preoperative chlorhexidine showers}

The efficacy of preoperative chlorhexidine showers is controversial. A 2015 Cochrane review of randomized controlled trials using preoperative chlorhexidine showers to prevent SSI in general was inconclusive [30]. However, in a 2019 retrospective study of thoracolumbar spine surgery, pre-surgery chlorhexidine shower was related to reduced odds of SSI [14,31]. In this study, patients were provided detailed instructions and were asked to shower with chlorhexidine soap at least 3 times before the surgery, with the last shower on the morning before the surgery [31]. The rationale for this measure is associated with the presence of a vast majority of causative pathogens in SSI originating from the skin flora [14]. This effect was not observed in patients undergoing spinal fusion, potentially owing to the longer operative time, underscoring the need to tailor SSI prevention to specific spinal procedures [31].

\section{Antimicrobial surgical preparation}

The surgical site can be prepared with either povidone-iodine or chlorhexidine. In a 2012 prospective randomized trial, there was no significant difference in skin contamination with the use of DuraPrep $(0.7 \%$ available iodine and $74 \%$ isopropyl alcohol; $3 \mathrm{M}$ Company, St. Paul, MN, USA) and ChloraPrep (2\% chlorhexidine gluconate and $70 \%$ isopropyl alcohol; CareFusion Inc., San Diego, CA, USA) [32]. However, multiple studies conducted thereafter have compared the efficacy of chlorhexidine to that of povidone-iodine, with chlorhexidine generally showing more favorable results [33]. This may be owing to the longer-lasting bactericidal effects of chlorhexidine on the skin [22]. In addition, intraoperative shaving of the surgical site with surgical clippers was reported to reduce the risk of SSI in one randomized trial [34]. A 2013 study by Pahys et al. [35] also demonstrated the efficacy of preparing the surgical site and plastic drapes with alcohol foam before the use of a surgical site preparation in reducing SSI in cervical spine surgery.

\section{Intraoperative Interventions}

\section{Antibiotics}

The North American Spine Society has developed evidence-based guidelines for antibiotic prophylaxis in spine surgery [36]. The use of antibiotic prophylaxis before spinal procedures reduces the risk of postoperative infection [37,38]. A meta-analysis by Barker [38] reported infection rates were $2.2 \%$ with the use of antibiotics, while the infection rate was $5.9 \%$ without the use of antibiotics. A randomized placebo-controlled trial by Petignat et al. [37] demonstrated an infection rate of $1.3 \%$ when patients received a $1.5 \mathrm{~g}$ dose of cefuroxime before lumbar laminotomy and discectomy for herniated disc and $2.8 \%$ when patients received the placebo.

First-generation cephalosporins, such as cephazolin, have demonstrated efficacy in numerous clinical trials [37,39-41]. A randomized trial by Rubinstein et al. [40] demonstrated a $4.3 \%$ wound infection rate in patients receiving $1 \mathrm{~g}$ of cefazolin about 2 hours preoperatively, while the placebo group had a $12.7 \%$ wound infection rate [40]. Clindamycin is an acceptable alternative for patients with penicillin or beta-lactam allergies. In recent years, the use of vancomycin has become popular; however, the risk of developing bacterial resistance should discourage the use of vancomycin unless patients test positive for MRSA colonization preoperatively [39].

The timing of antibiotic administration is as important as the selection of the correct antibiotic when trying to prevent a postoperative infection. Optimal time must be given to allow sufficient accumulation of the antibiotic in the serum and tissues before making the incision. A multicenter study by Steinberg et al. [41] showed that the risk of infection increased as the time between antibiotic infusion and incision increased. They showed that the administration of antibiotics, with short infusion times, within 30 minutes before the incision reduced the infection risk as compared to that with the administration of antibiotics within 31-60 minutes before the incision [41]. For longer spinal procedures, redosing of antibiotics is generally recommended. Although no studies have directly compared redosing to not redosing, some studies have used redosing protocols in their samples [42-44]. For longer procedures, it is generally acceptable to redose antibiotics, such as cefazolin, every 3-4 hours to maintain therapeutic levels throughout the procedure [36]. 


\section{Intraoperative warming}

Intraoperative core temperatures can alter peripheral circulation and tissue oxygenation. Intraoperative hypoxia is known to increase the risk of wound infection in animal and human studies [45-47]. Mild hypothermia during anesthesia lowers immune resistance to infectious bacteria, such as E. coli and S. aureus in animal models $[45,46]$. Increased wound infection risk could result from vasoconstriction induced by hypothermia, leading to increased tissue hypoxia, causing the surgical site to be more susceptible to infection. Hypoxia induced by vasoconstriction may also impair wound healing because scar formation requires hydroxylation reactions that are dependent on oxygen tension [47]. In contrast, mild hyperthermia is not protective during anesthesia [46].

Intraoperative normothermia is known to be most effective in reducing the risk of developing an SSI. A randomized clinical trial that compared intraoperative hypothermia with normothermia in patients undergoing colorectal surgery has shown decreased surgical infection rates and shorter hospitalizations in patients receiving intraoperative normothermia [47]. A Cochrane review demonstrated reduced rates of SSI in patients undergoing abdominal surgery when active warming systems were used [48]. Thus, forced-air warming systems should be regularly used in patients who are undergoing spine surgery to maintain intraoperative normothermia and reduce the risk of SSI.

\section{Vancomycin powder}

Intravenous vancomycin can be used as an effective prophylactic agent against MRSA infections; however, its use is not routinely recommended owing to concerns regarding the emergence of vancomycin-resistant organisms and lack of gram-negative coverage $[49,50]$. However, vancomycin powder is a beneficial alternative. Vancomycin powder can be easily applied in the wound bed, is inexpensive, and is able to achieve high local concentrations while minimizing serum concentrations of the drug [5154].

A meta-analysis that examined the effectiveness of local vancomycin powder for decreasing SSIs found that the administration of vancomycin powder was significantly protective against SSIs, deep incisional SSIs, and S. aureus SSIs after spinal procedures [51]. Protective effects of in- trawound vancomycin powder against SSIs have also been supported by systematic reviews [55-57]. However, two studies have shown no significant reduction in the SSI incidence with the use of intrasite vancomycin powder and do not support the use of local vancomycin powder in spine surgery [58,59].

Owing to the local application and undetectable levels of serum vancomycin [60], adverse effects of local vancomycin powder are rarely reported [49]. Although the adverse effect profile of local vancomycin powder may be minimal, the widespread use of vancomycin powder in spine surgery may promote the development of vancomycin-resistant organisms. Furthermore, the routine use of a broad-spectrum antibiotic that provides mostly grampositive coverage may result in an altered SSI microbial profile, predominated by gram-negative species, anaerobic bacteria, or polymicrobial infections $[49,50,61,62]$. In a retrospective study by Ghobrial et al. [50], 60.7\% of the positive cultures contained gram-negative organisms in the vancomycin powder group compared to $21 \%$ in the non-vancomycin powder control group.

\section{Postoperative Interventions}

\section{Intrawound drains}

Wound draining is a commonly used method used for promoting wound healing and preventing SSI [63], although their use in spine surgery is controversial [64]. Wound drains remove excess post-surgical fluids in the epidural and subfascial spaces and prevent hematoma and dead space formation $[64,65]$. Dead space cavities may accumulate serous fluids that hamper the wound healing process or promote subsequent infections [66]. However, drains prolong hospital stay, increase patient discomfort, and may result in increased need for blood transfusions. Further, some argue that wound drains increase the risk of postoperative infection by retaining a connection to the outside environment $[64,65,67,68]$. For example, prolonged post-surgical drain retention was reported to increase the risk for deep SSI after spine surgery, suggesting that expedited drain discharge may reduce the SSI risk SSI [67]. Positive drain tip culture has been proposed as an early prognosticator of SSI; however, a 2016 retrospective study of drain culture reported a $0 \%$ sensitivity of wound tip culture for SSI, with no correlation between SSI and drain tip culture [69]. A 2016 systematic review of 
wound drains in non-complex lumbar surgery identified no significant difference between the incidence of postoperative spinal hematomas or wound infections in patients with drains and in those without drains [64]. Similarly, a prospective randomized study of closed wound suction drainage vs. no drainage in adolescent idiopathic scoliosis patients undergoing posterior fusion did not identify any advantage of deep wound drainage with respect to infection prevention [70].

In contrast, a multicenter retrospective study of drains in cervical spine surgery suggested that drains may play a role in preventing SSI in this population [71]. It is noteworthy that infections and postoperative hematomas carry greater morbidity after cervical spine surgery, underscoring the importance of a tailored approach to SSI prevention [71]. In a similar manner, high vacuum closed suction drainage was efficacious in treating late-onset deep SSI after instrumented spinal surgery [72]. However, preventative closed suction drainage did not lower the risk of infection after spine surgery in a 2016 meta-analysis [73]. This suggests that wound drains are most helpful in SSI management when inserted after SSI debridement rather than as a universal preventative measure. A retrospective study that compared one vs. two drains in treating SSI after lumbar spine surgery found that a single drain was associated with better clinical outcomes and a shorter hospital stay [74].

\section{Negative pressure wound therapy}

Deep wound vacuum assisted closure (VAC; Kinetic Concepts Inc., San Antonio, TX, USA) is another important postoperative tool for both, managing and preventing SSI. VACs are a form of negative pressure wound therapy (NPWT) that uses negative pressure to close dead spaces, maintain the sterile field, improve wound vascularity, increase skin perfusion, and clear infections [75-77]. To place a VAC, the surgeon inserts a foam dressing that has been cut into the shape of the wound, allowing it to compress along the complete surface area of the lesion. A drainage tube is located above the foam that is further covered by another layer of foam along with the provided adhesive transparent membrane [78]. After forming a proper seal, the distal end of the drain is connected to a vacuum that absorbs air within the foam block, causing the ends of the wound to draw inward while allowing the fluid within the wound to be absorbed by the foam and be transported to a disposable unit [78]. Earlier studies on the use of VACs in spine surgery have focused on management, rather than the prevention of SSI [76]. Several small-scale $(\mathrm{n}<50)$ retrospective studies have shown the efficacy of NPWT in managing SSI after spine surgery [75,79-82]. More recently, a 2014 retrospective cohort study demonstrated a reduced incidence of SSI in patients undergoing long-segment thoracolumbar fusion [82]. A 2020 retrospective cohort study of a single surgeon suggested that NPWT reduces the risk of dehiscence and SSI when used in a high-risk population (patients with neoplasm or infection, long incisions, long fusion constructs, intraoperative durotomy, etc.) [77]. It is noteworthy that NPWT was demonstrated safe in a population of patients with deep SSI and durotomy: it did not result in CSFrelated complications [83].

\section{Surgical incision dressing (Silver, Aquaphor, Prevena)}

There is some ambiguity with respect to the efficacy of wound dressings in lowering the prevalence of SSI. In terms of their general function, wound dressings are meant to provide structural and protective support in wound closure and absorb extraneous exudates [84]. Occlusive dressings can also provide a sterile barrier for the wound and have been demonstrated to reduce the rate of wound complication (infection, significant erythema and drainage, blistered skin, need for revision) in spine surgery [68]. Silver-impregnated dressing is one of several wound dressing options that are available. While silver has antimicrobial properties, it can compromise keratinocyte and fibroblast activity by inducing cytotoxic effects [84]. Further studies are needed to characterize the efficacy of silver wound dressings because recent systematic reviews have been inconclusive [84-86]. However, a 2007 retrospective study of silver dressings versus standard gauze showed a reduction in the risk of both, deep and superficial SSI [87]. Several randomized controlled trials and reviews have shown that silver in its nanocrystalline form improves the outcomes in wounds with high microbial counts and foul site odors when applied within the first 2 weeks of infection [84]. In addition, silver dressings do not require frequent dressing maintenance; this helps reduce pain [70]. However, when the wound becomes cleaner, silver dressings should be discontinued to prevent any cytotoxic effects [84].

Aquaphor dressing (Beiersdorf Inc., Wilton, CT, USA) 
is another wound dressing that has shown promise in SSI prevention. Aquaphor is a non-adherent, petrolatumcomposed, multipurpose dressing made of acetate substrates with numerous dermatologic benefits [88]. While the literature on the use of Aquaphor in surgical care is sparse, Aquaphor increased the rate of wound contraction and epithelialization, with notably smaller wound size by day 10, in a rat model [88]. In addition, a large randomized trial of ambulatory surgery patients demonstrated equivalence between petrolatum, the chief component of Aquaphor, and bacitracin in this population [89]. However, Aquaphor can potentially cause allergic dermatitislike symptoms, such as erythema, edema, postoperative blisters, itchiness, and oozing at the operative site. This has often made the use of Aquaphor in infection treatment controversial owing to concerns that the dermatitis symptoms may impede wound healing [90]. However, when combined with antibiotic therapy, Aquaphor has demonstrated positive preliminary results, although further studies on its use in SSI are necessary [90].

The novel Prevena Incision Management System (3M Company) is a unique surgical dressing that implements NPWT [91]. This system has been commercially available since 2010 and protects the surgical incision from contamination by using negative pressure suction to hold the edges of the edges together and remove the fluid and infected agents [92]. Compared to standard VAC devices, Prevena allows uniform pressure across the lesion by implementing an additional $2.5-\mathrm{cm}$ foam filler [92]. In case reports, as compared to the use of gauze dressing, the use of Prevena has lowered postoperative pain, reduced hematoma occurrence, and shortened the wound healing time [92]. In a retrospective study, Prevena reduced the incidence of groin wound infection in patients after vascular surgery as compared to that with standard absorbent dressing and skin adhesive [93]. Prevena has also been demonstrated to reduce the need for revision surgery in deep SSI after sternotomy, suggesting that it is applicable in both, SSI management and prevention [94]. Prevena is cost effective because it reduces the need for daily dressing changes [92]. In 2019, the National Institute for Clinical Excellence concluded that Prevena is the most effective technique for reducing postoperative surgical infections. Till date, it remains the first and only NPWT device that is approved by the Food and Drug Administration for reducing SSI prevalence [92].

Table 1. Overview of the preoperative, intraoperative, and postoperative interventions for surgical site infection

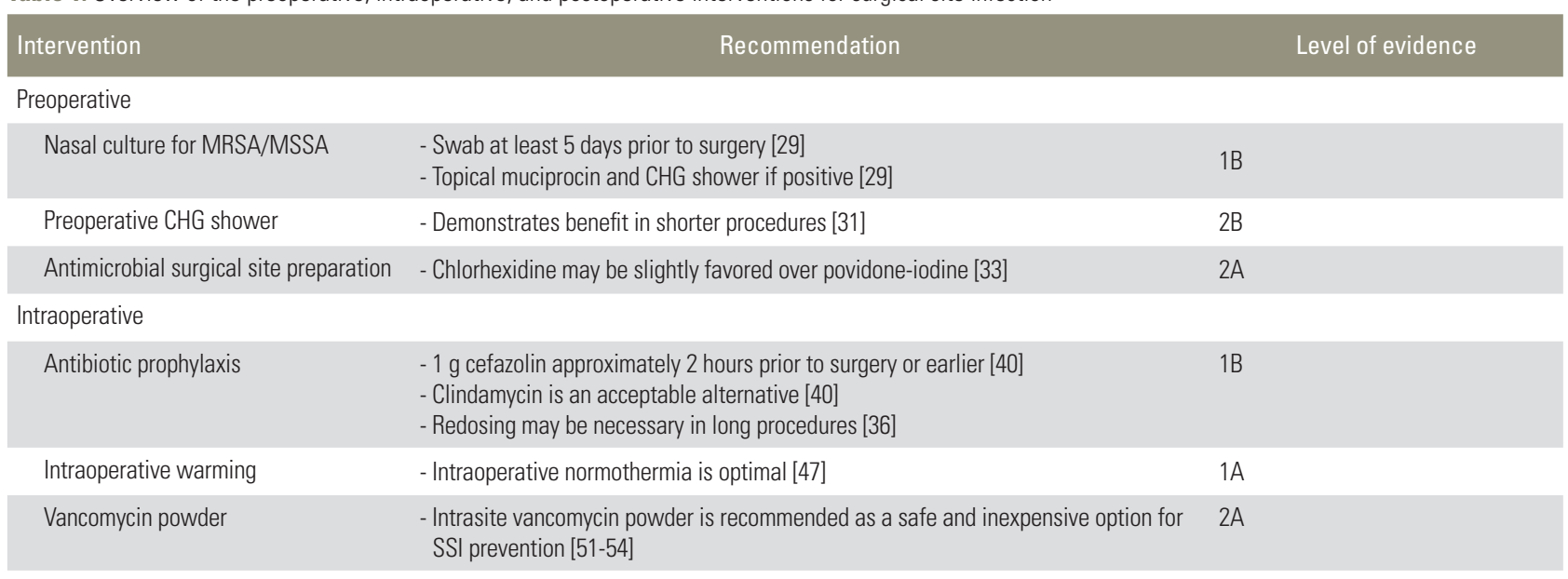

Postoperative

\begin{tabular}{lll}
\hline Wound drains & - Limited evidence for SSI prevention [64] & 2A \\
\hline Negative pressure wound therapy & - May be useful in SSI management [74] & - Evidence for efficacy in SSI management [76] \\
& - Emerging evidence for efficacy in SSI prevention [83] & 2A \\
\hline Traditional wound dressings & $\begin{array}{l}\text { - Silver and Aquaphor dressings both have antimicrobrial properties and may acceler- } \\
\text { ate wound healing [88,90] }\end{array}$ & 1B; 2A \\
\hline Prevena wound management system & - Unique dressing that reduces SSI prevalence [93] & More studies needed in spine surgery \\
\hline
\end{tabular}

MRSA, Methicillin-resistant Staphylococcus aureus; MSSA, Methicillin-sensitive Staphylococcus aureus; CHG, chlorhexidine; SSI, surgical site infection. 


\section{Conclusions}

SSI is a common and potentially devastating complication following spine surgery that is associated with increased morbidity, mortality, and healthcare costs. While some risk factors for SSI after spine surgery remain controversial, diabetes, elevated BMI, high ASA score, posterior approach, durotomy, surgery after trauma, and use of instrumentation are commonly reported. Numerous strategies to prevent and manage SSI in the preoperative, intraoperative, and postoperative period are available (Table 1), although their use should be tailored to individual patients and procedures. The SSI risk can be mitigated with optimal preoperative management of chronic diseases, preoperative MRSA and MSSA swabs, and chlorhexidine showers. During surgery, the use of chlorhexidine-ethanol surgical site preparation, appropriate antibiotic prophylaxis, wound irrigation, and vancomycin powder can further prevent SSIs. While the use of drains in spine surgery is controversial and does not appear to play a significant role in reducing the SSI incidence, NPWT is a promising approach for both, the prevention of SSI in high-risk populations and the management of SSI. Wound dressings can include silver or Aquaphor that possess antimicrobial properties and promote wound healing. Future studies are needed on the efficacy of different wound dressings in spine surgery. Prevena is a promising NPWT surgical wound dressing; broader studies on its efficacy in spine surgery may allow widespread implementation. A reliable risk calculator for SSI after spine surgery would help tailor infection prevention and wound management approaches for individual patients. SSI prevention and wound management should strike a balance between universal protocols to mitigate human error and a tailored approach that considers the individual risk factors of each patient and procedure.

\section{Conflict of Interest}

No potential conflict of interest relevant to this article was reported.

\section{References}

1. Lee MJ, Cizik AM, Hamilton D, Chapman JR. Predicting surgical site infection after spine surgery: a validated model using a prospective surgical registry.
Spine J 2014;14:2112-7.

2. Lonjon G, Dauzac C, Fourniols E, et al. Early surgical site infections in adult spinal trauma: a prospective, multicentre study of infection rates and risk factors. Orthop Traumatol Surg Res 2012;98:788-94.

3. Saeedinia S, Nouri M, Azarhomayoun A, et al. The incidence and risk factors for surgical site infection after clean spinal operations: a prospective cohort study and review of the literature. Surg Neurol Int 2015;6:154.

4. Zhou J, Wang R, Huo X, Xiong W, Kang L, Xue Y. Incidence of surgical site infection after spine surgery: a systematic review and meta-analysis. Spine (Phila $\mathrm{Pa}$ 1976) 2020;45:208-16.

5. Veeravagu A, Patil CG, Lad SP, Boakye M. Risk factors for postoperative spinal wound infections after spinal decompression and fusion surgeries. Spine (Phila Pa 1976) 2009;34:1869-72.

6. Koutsoumbelis S, Hughes AP, Girardi FP, et al. Risk factors for postoperative infection following posterior lumbar instrumented arthrodesis. J Bone Joint Surg Am 2011;93:1627-33.

7. Kuhns BD, Lubelski D, Alvin MD, et al. Cost and quality of life outcome analysis of postoperative infections after subaxial dorsal cervical fusions. J Neurosurg Spine 2015;22:381-6.

8. Bernatz JT, Anderson PA. Thirty-day readmission rates in spine surgery: systematic review and metaanalysis. Neurosurg Focus 2015;39:E7.

9. Yeramaneni S, Robinson C, Hostin R. Impact of spine surgery complications on costs associated with management of adult spinal deformity. Curr Rev Musculoskelet Med 2016;9:327-32.

10. Atkinson RA, Jones A, Ousey K, Stephenson J. Management and cost of surgical site infection in patients undergoing surgery for spinal metastasis. J Hosp Infect 2017;95:148-53.

11. Blumberg TJ, Woelber E, Bellabarba C, Bransford R, Spina N. Predictors of increased cost and length of stay in the treatment of postoperative spine surgical site infection. Spine J 2018;18:300-6.

12. Godil SS, Parker SL, O’Neill KR, Devin CJ, McGirt MJ. Comparative effectiveness and cost-benefit analysis of local application of vancomycin powder in posterior spinal fusion for spine trauma: clinical article. J Neurosurg Spine 2013;19:331-5.

13. Agarwal N, Agarwal P, Querry A, et al. Implementa- 
tion of an infection prevention bundle and increased physician awareness improves surgical outcomes and reduces costs associated with spine surgery. J Neurosurg Spine 2018;29:108-14.

14. Deng H, Chan AK, Ammanuel S, et al. Risk factors for deep surgical site infection following thoracolumbar spinal surgery. J Neurosurg Spine 2019;32:292301.

15. Meng F, Cao J, Meng X. Risk factors for surgical site infections following spinal surgery. J Clin Neurosci 2015;22:1862-6.

16. Schuster JM, Rechtine G, Norvell DC, Dettori JR. The influence of perioperative risk factors and therapeutic interventions on infection rates after spine surgery: a systematic review. Spine (Phila Pa 1976) 2010;35(9 Suppl):S125-37.

17. Radcliff KE, Neusner AD, Millhouse PW, et al. What is new in the diagnosis and prevention of spine surgical site infections. Spine J 2015;15:336-47.

18. Ogihara S, Yamazaki T, Shiibashi M, et al. Risk factor analysis of deep surgical site infection after posterior instrumented fusion surgery for spinal trauma: a multicenter observational study. World Neurosurg 2020;134:e524-9.

19. Abdallah DY, Jadaan MM, McCabe JP. Body mass index and risk of surgical site infection following spine surgery: a meta-analysis. Eur Spine J 2013;22:2800-9.

20. Anderson PA, Savage JW, Vaccaro AR, et al. Prevention of surgical site infection in spine surgery. Neurosurgery 2017;80:S114-23.

21. Blood AG, Sandoval MF, Burger E, Halverson-Carpenter K. Risk and protective factors associated with surgical infections among spine patients. Surg Infect (Larchmt) 2017;18:234-49.

22. Olsen MA, Nepple JJ, Riew KD, et al. Risk factors for surgical site infection following orthopaedic spinal operations. J Bone Joint Surg Am 2008;90:62-9.

23. Butler JS, Wagner SC, Morrissey PB, et al. Strategies for the prevention and treatment of surgical site infection in the lumbar spine. Clin Spine Surg 2018;31:323-30.

24. McGirt MJ, Parker SL, Lerner J, Engelhart L, Knight T, Wang MY. Comparative analysis of perioperative surgical site infection after minimally invasive versus open posterior/transforaminal lumbar interbody fusion: analysis of hospital billing and discharge data from 5,170 patients. J Neurosurg Spine 2011;14:771-8.
25. Haleem A, Chiang HY, Vodela R, et al. Risk factors for surgical site infections following adult spine operations. Infect Control Hosp Epidemiol 2016;37:145867.

26. Ning J, Wang J, Zhang S, Sha X. Nasal colonization of Staphylococcus aureus and the risk of surgical site infection after spine surgery: a meta-analysis. Spine J 2020;20:448-56.

27. Higgins M, Bommireddy R, Shivji F, Al-Shukri J, Billson J. Impact of MSSA screening on rates of surgical site infection following lumbar spine surgery. Eur Spine J 2018;27:2457-62.

28. Thakkar V, Ghobrial GM, Maulucci CM, et al. Nasal MRSA colonization: impact on surgical site infection following spine surgery. Clin Neurol Neurosurg 2014;125:94-7.

29. Bode LG, Kluytmans JA, Wertheim HF, et al. Preventing surgical-site infections in nasal carriers of Staphylococcus aureus. N Engl J Med 2010;362:9-17.

30. Webster J, Osborne S. Preoperative bathing or showering with skin antiseptics to prevent surgical site infection. Cochrane Database Syst Rev 2015;(2):CD004985.

31. Chan AK, Ammanuel SG, Chan AY, et al. Chlorhexidine showers are associated with a reduction in surgical site infection following spine surgery: an analysis of 4266 consecutive surgeries. Neurosurgery 2019;85:817-26.

32. Savage JW, Weatherford BM, Sugrue PA, et al. Efficacy of surgical preparation solutions in lumbar spine surgery. J Bone Joint Surg Am 2012;94:490-4.

33. Lee I, Agarwal RK, Lee BY, Fishman NO, Umscheid CA. Systematic review and cost analysis comparing use of chlorhexidine with use of iodine for preoperative skin antisepsis to prevent surgical site infection. Infect Control Hosp Epidemiol 2010;31:1219-29.

34. Celik SE, Kara A. Does shaving the incision site increase the infection rate after spinal surgery? Spine (Phila Pa 1976) 2007;32:1575-7.

35. Pahys JM, Pahys JR, Cho SK, et al. Methods to decrease postoperative infections following posterior cervical spine surgery. J Bone Joint Surg Am 2013;95:549-54.

36. Shaffer WO, Baisden JL, Fernand R, Matz PG; North American Spine Society. An evidence-based clinical guideline for antibiotic prophylaxis in spine surgery. Spine J 2013;13:1387-92.

37. Petignat C, Francioli P, Harbarth S, et al. Cefuroxime 
prophylaxis is effective in noninstrumented spine surgery: a double-blind, placebo-controlled study. Spine (Phila Pa 1976) 2008;33:1919-24.

38. Barker FG 2nd. Efficacy of prophylactic antibiotic therapy in spinal surgery: a meta-analysis. Neurosurgery 2002;51:391-400.

39. Appelbaum PC. The emergence of vancomycin-intermediate and vancomycin-resistant Staphylococcus aureus. Clin Microbiol Infect 2006;12 Suppl 1:16-23.

40. Rubinstein E, Findler G, Amit P, Shaked I. Perioperative prophylactic cephazolin in spinal surgery: a double-blind placebo-controlled trial. J Bone Joint Surg Br 1994;76:99-102.

41. Steinberg JP, Braun BI, Hellinger WC, et al. Timing of antimicrobial prophylaxis and the risk of surgical site infections: results from the trial to reduce antimicrobial prophylaxis errors. Ann Surg 2009;250:10-6.

42. Riley LH 3rd. Prophylactic antibiotics for spine surgery: description of a regimen and its rationale. J South Orthop Assoc 1998;7:212-7.

43. Hellbusch LC, Helzer-Julin M, Doran SE, et al. Single-dose vs multiple-dose antibiotic prophylaxis in instrumented lumbar fusion: a prospective study. Surg Neurol 2008;70:622-7.

44. Kakimaru H, Kono M, Matsusaki M, Iwata A, Uchio Y. Postoperative antimicrobial prophylaxis following spinal decompression surgery: is it necessary? J Orthop Sci 2010;15:305-9.

45. Sheffield CW, Sessler DI, Hunt TK, Scheuenstuhl H. Mild hypothermia during halothane-induced anesthesia decreases resistance to Staphylococcus aureus dermal infection in guinea pigs. Wound Repair Regen 1994;2:48-56.

46. Sheffield CW, Sessler DI, Hunt TK. Mild hypothermia during isoflurane anesthesia decreases resistance to E. coli dermal infection in guinea pigs. Acta Anaesthesiol Scand 1994;38:201-5.

47. Kurz A, Sessler DI, Lenhardt R. Perioperative normothermia to reduce the incidence of surgicalwound infection and shorten hospitalization: study of Wound Infection and Temperature Group. N Engl J Med 1996;334:1209-15.

48. Madrid E, Urrutia G, Roque i Figuls M, et al. Active body surface warming systems for preventing complications caused by inadvertent perioperative hypothermia in adults. Cochrane Database Syst Rev 2016;4:CD009016.
49. Ghobrial GM, Cadotte DW, Williams K Jr, Fehlings MG, Harrop JS. Complications from the use of intrawound vancomycin in lumbar spinal surgery: a systematic review. Neurosurg Focus 2015;39:E11.

50. Ghobrial GM, Thakkar V, Andrews E, et al. Intraoperative vancomycin use in spinal surgery: single institution experience and microbial trends. Spine (Phila Pa 1976) 2014;39:550-5.

51. Chiang HY, Herwaldt LA, Blevins AE, Cho E, Schweizer ML. Effectiveness of local vancomycin powder to decrease surgical site infections: a meta-analysis. Spine J 2014;14:397-407.

52. Theologis AA, Demirkiran G, Callahan M, Pekmezci M, Ames C, Deviren V. Local intrawound vancomycin powder decreases the risk of surgical site infections in complex adult deformity reconstruction: a cost analysis. Spine (Phila Pa 1976) 2014;39:1875-80.

53. Sweet FA, Roh M, Sliva C. Intrawound application of vancomycin for prophylaxis in instrumented thoracolumbar fusions: efficacy, drug levels, and patient outcomes. Spine (Phila Pa 1976) 2011;36:2084-8.

54. Heller A, McIff TE, Lai SM, Burton DC. Intrawound vancomycin powder decreases staphylococcal surgical site infections after posterior instrumented spinal arthrodesis. J Spinal Disord Tech 2015;28:E584-9.

55. Evaniew N, Khan M, Drew B, Peterson D, Bhandari $\mathrm{M}$, Ghert M. Intrawound vancomycin to prevent infections after spine surgery: a systematic review and meta-analysis. Eur Spine J 2015;24:533-42.

56. Bakhsheshian J, Dahdaleh NS, Lam SK, Savage JW, Smith ZA. The use of vancomycin powder in modern spine surgery: systematic review and meta-analysis of the clinical evidence. World Neurosurg 2015;83:81623.

57. Kang DG, Holekamp TF, Wagner SC, Lehman RA Jr. Intrasite vancomycin powder for the prevention of surgical site infection in spine surgery: a systematic literature review. Spine J 2015;15:762-70.

58. Tubaki VR, Rajasekaran S, Shetty AP. Effects of using intravenous antibiotic only versus local intrawound vancomycin antibiotic powder application in addition to intravenous antibiotics on postoperative infection in spine surgery in 907 patients. Spine (Phila Pa 1976) 2013;38:2149-55.

59. Martin JR, Adogwa O, Brown CR, et al. Experience with intrawound vancomycin powder for spinal deformity surgery. Spine (Phila Pa 1976) 2014;39:177- 
84.

60. Gans I, Dormans JP, Spiegel DA, et al. Adjunctive vancomycin powder in pediatric spine surgery is safe. Spine (Phila Pa 1976) 2013;38:1703-7.

61. Adogwa O, Elsamadicy AA, Sergesketter A, et al. Prophylactic use of intraoperative vancomycin powder and postoperative infection: an analysis of microbiological patterns in 1200 consecutive surgical cases. J Neurosurg Spine 2017;27:328-34.

62. Hey HW, Thiam DW, Koh ZS, et al. Is intraoperative local vancomycin powder the answer to surgical site infections in spine surgery? Spine (Phila Pa 1976) 2017;42:267-74.

63. Von Eckardstein KL, Dohmes JE, Rohde V. Use of closed suction devices and other drains in spinal surgery: results of an online, Germany-wide questionnaire. Eur Spine J 2016;25:708-15.

64. Zijlmans JL, Buis DR, Verbaan D, Vandertop WP. Wound drains in non-complex lumbar surgery: a systematic review. Bone Joint J 2016;98-B:984-9.

65. Patel SB, Griffiths-Jones W, Jones CS, et al. The current state of the evidence for the use of drains in spinal surgery: systematic review. Eur Spine J 2017;26:2729-38.

66. Fay MF. Drainage systems: their role in wound healing. AORN J 1987;46:442-55.

67. Pennington Z, Lubelski D, Molina C, Westbroek EM, Ahmed AK, Sciubba DM. Prolonged post-surgical drain retention increases risk for deep wound infection after spine surgery. World Neurosurg 2019;130:e846-53.

68. Andrew Glennie R, Dea N, Street JT. Dressings and drains in posterior spine surgery and their effect on wound complications. J Clin Neurosci 2015;22:10817.

69. Yamada T, Yoshii T, Egawa S, et al. Drain tip culture is not prognostic for surgical site infection in spinal surgery under prophylactic use of antibiotics. Spine (Phila Pa 1976) 2016;41:1179-84.

70. Ovadia D, Drexler M, Kramer M, Herman A, Lebel DE. Closed wound subfascial suction drainage in posterior fusion surgery for adolescent idiopathic scoliosis: a prospective randomized control study. Spine (Phila Pa 1976) 2019;44:377-83.

71. Herrick DB, Tanenbaum JE, Mankarious M, et al. The relationship between surgical site drains and reoperation for wound-related complications following posterior cervical spine surgery: a multicenter retrospective study. J Neurosurg Spine 2018;29:628-34.

72. Yin D, Liu B, Chang Y, Gu H, Zheng X. Management of late-onset deep surgical site infection after instrumented spinal surgery. BMC Surg 2018;18:121.

73. Liu Y, Li Y, Miao J. Wound drains in posterior spinal surgery: a meta-analysis. J Orthop Surg Res 2016;11:16.

74. Chen YC, Zhang L, Li EN, et al. One or two drains for the treatment of surgical site infections after lumbar spine surgery. World Neurosurg 2018;116:e18-25.

75. Alcala-Marquez C, Torrealba R, Mehbod AA, Dawson JM, Dressel TD, Transfeldt EE. Recurrent surgical site infections in the spine after treatment with the vacuum-assisted closure (VAC) system. Clin Spine Surg 2018;31:351-5.

76. Ousey KJ, Atkinson RA, Williamson JB, Lui S. Negative pressure wound therapy (NPWT) for spinal wounds: a systematic review. Spine J 2013;13:1393405.

77. Naylor RM, Gilder HE, Gupta N, et al. Effects of negative pressure wound therapy on wound dehiscence and surgical site infection following instrumented spinal fusion surgery: a single surgeon's experience. World Neurosurg 2020;137:e257-62.

78. Yadav S, Rawal G, Baxi M. Vacuum assisted closure technique: a short review. Pan Afr Med J 2017;28:246.

79. Kale M, Padalkar P, Mehta V. Vacuum-assisted closure in patients with post-operative infections after instrumented spine surgery: a series of 12 cases. J Orthop Case Rep 2017;7:95-100.

80. van Rhee MA, de Klerk LW, Verhaar JA. Vacuumassisted wound closure of deep infections after instrumented spinal fusion in six children with neuromuscular scoliosis. Spine J 2007;7:596-600.

81. Canavese F, Marengo L, Corradin M, et al. Deep postoperative spine infection treated by negative pressure therapy in patients with progressive spinal deformities. Arch Orthop Trauma Surg 2018;138:463-9.

82. Adogwa O, Fatemi P, Perez E, et al. Negative pressure wound therapy reduces incidence of postoperative wound infection and dehiscence after long-segment thoracolumbar spinal fusion: a single institutional experience. Spine J 2014;14:2911-7.

83. Ridwan S, Grote A, Simon M. Safety and efficacy of negative pressure wound therapy for deep spinal wound infections after dural exposure, durotomy, or 
intradural surgery. World Neurosurg 2020;134:e62430.

84. Khansa I, Schoenbrunner AR, Kraft CT, Janis JE. Silver in wound care-friend or foe?: a comprehensive review. Plast Reconstr Surg Glob Open 2019;7:e2390.

85. Abboud EC, Settle JC, Legare TB, Marcet JE, Barillo DJ, Sanchez JE. Silver-based dressings for the reduction of surgical site infection: review of current experience and recommendation for future studies. Burns 2014;40 Suppl 1:S30-9.

86. Leaper DJ. Silver dressings: their role in wound management. Int Wound J 2006;3:282-94.

87. Epstein NE. Do silver-impregnated dressings limit infections after lumbar laminectomy with instrumented fusion? Surg Neurol 2007;68:483-5.

88. Little JW, Bickley HC, Daugherty WB, Bickley C. Effect of aquaphor ointment on wound healing. J Dent Res 1972;51:1672-4.

89. Smack DP, Harrington AC, Dunn C, et al. Infection and allergy incidence in ambulatory surgery patients using white petrolatum vs bacitracin ointment: a randomized controlled trial. JAMA 1996;276:972-7.
90. Tong QJ, Hammer KD, Johnson EM, Zegarra M, Goto M, Lo TS. A systematic review and meta-analysis on the use of prophylactic topical antibiotics for the prevention of uncomplicated wound infections. Infect Drug Resist 2018;11:417-25.

91. Hyldig N, Birke-Sorensen H, Kruse M, et al. Metaanalysis of negative-pressure wound therapy for closed surgical incisions. Br J Surg 2016;103:477-86.

92. Huan S, Tay L. Four curious cases of closed-incision negative pressure therapy (ciNPT). Cureus 2020;12:e8193.

93. Matatov T, Reddy KN, Doucet LD, Zhao CX, Zhang WW. Experience with a new negative pressure incision management system in prevention of groin wound infection in vascular surgery patients. J Vasc Surg 2013;57:791-5.

94. Nickl S, Steindl J, Langthaler D, et al. First experiences with incisional negative pressure wound therapy in a high-risk poststernotomy patient population treated with pectoralis major muscle flap for deep sternal wound infection. J Reconstr Microsurg 2018;34:1-7. 